\title{
3D Modeling of One and Two Component Gas Flow in Fibrous Microstructures in Fuel Cells by Using the Lattice-Boltzmann Method
}

\author{
Jan P. Brinkmann ${ }^{\mathrm{a}}$, Dieter Froning ${ }^{\mathrm{a}}$, Uwe Reimer ${ }^{\mathrm{a}}$, Volker Schmidt ${ }^{\mathrm{b}}$, Werner Lehnert ${ }^{\mathrm{a}}$, \\ Detlef Stolten ${ }^{\mathrm{a}}$ \\ ${ }^{a}$ Forschungszentrum Jülich GmbH, Institute of Energy and Climate Research, IEK-3: \\ Fuel Cells, 52425 Jülich, Germany \\ ${ }^{\mathrm{b}}$ Institute of Stochastics, Ulm University, 89069 Ulm, Germany
}

\begin{abstract}
In fuel cells, a homogeneous distribution of gas flow is desirable for optimal performance. The gas diffusion layer (GDL) often used in PEM-like fuel cells is one of the key elements responsible for a uniform distribution under channels and ribs. To assess this ability of GDL-materials, characteristic numbers, e.g. the permeability, are often introduced. In this paper, we simulate one and two component gas flow through a virtual GDL material under operating conditions of a HT-PEFC. We observe the influence of discretization and viscosity choice on the macroscopic output of the Lattice-Boltzmann algorithm we apply. To achieve this, we first study their effects on empty square channels and finally transfer our interpretation to the output of a GDL simulation.
\end{abstract}

\section{Introduction}

The electrochemical reaction in a fuel cell takes place at the three-phase boundary of its electrodes. Hence, the gas supply of this area is crucial for its effectiveness. In a PEMlike fuel cell, this supply is often supported by the Gas Diffusion Layer (GDL), which is connected to the electrode either directly or separated through a Microporous Layer (MPL). On both sides of this Membrane Electrode Assembly (MEA), gas channels of the bipolar plates provide the GDL with gas. The GDL's purpose is then to distribute the gas evenly over the full surface of the electrode under channels and ribs of the bipolar plates. Its permeability determines the quality of both the horizontal and the vertical gas supply at the electrode's surface.

Although all the single elements function as one cell, their geometrical structures are remarkably distinct and range from the $\mathrm{mm}$ scale (flow fields) over $\mu \mathrm{m}$ (GDL) to $\mathrm{nm}$ (electrode). A fuel cell model focussing on the $\mathrm{mm}$ scale often leads to a simplification or parameterization of the low scale regions. In contrast, models of microscopic regions are often limited in size and the simulated region does not reach the macroscopic size of a fuel cell. The dimension of the simulated region also influences the choice of the model. In order to simulate fluid flow in stacks for example, CFD simulations are often used (1). On the nanometer scale, implementations of continuum models are suitable options (2).

In this paper, the modeling concept and results of one and two component gas flow through the GDL is presented. The implemented boundary conditions are based on the operating conditions of a High-Temperature Polymer Electrolyte Fuel Cell (HT-PEFC). The stochastic model of the paper based GDL that we use in our simulations was developed by Thiedmann et al. $(3,4)$. In their model, the randomly arranged fibers are virtually reconstructed by statistical methods. In this virtual material, we apply the 
Lattice-Boltzmann method to simulate gas transport $(5,6)$. The combination of these two approaches enables us to dissolve the material to the microscopic scale in the simulation. The use of the Lattice-Boltzmann method makes the implementation of an unequal distribution of fibers possible and beyond that massively favors the parallelization of computer code. The results from the simulation are macroscopic density and velocity fields of the gas. For the analysis, we calculate characteristic numbers (permeability, tortuosity) that connect these results with the implemented fuel cell boundary conditions.

Before simulating fluid flow in the complex GDL structure, we start with the simulation of an empty square channel. Besides validation issues, this simulation is particularly done to analyze the influence of the discretization $\delta x$ and the collision parameter $\omega$ on the physical output. A similar approach was successfully chosen in (7). In a second step, our methods are applied on the virtual GDL. Possible influences caused by the discretization and the collision parameter are identified. We can use the characteristic numbers as a first evaluation of how well the modeled GDL serves its purpose of a homogenous distribution of gas at the electrode's surface. Finally, the comparison of the simulated permeability with experimental results leads to a first validation of our combined models.

\section{Methods and Models}

\section{$\underline{\text { GDL Model }}$}

In this section, we present a brief description of the GDL model. The idea of the model is to virtually rebuild a paper type Toray material, which may be used in PEM like fuel cells as a gas diffusion layer. In Figure 1 we illustrate a 2D section of this material. It consists of irregularly arranged straight fibers. In the manufacturing process these fibers are piled in layers on top of each other, held together by a binder material.

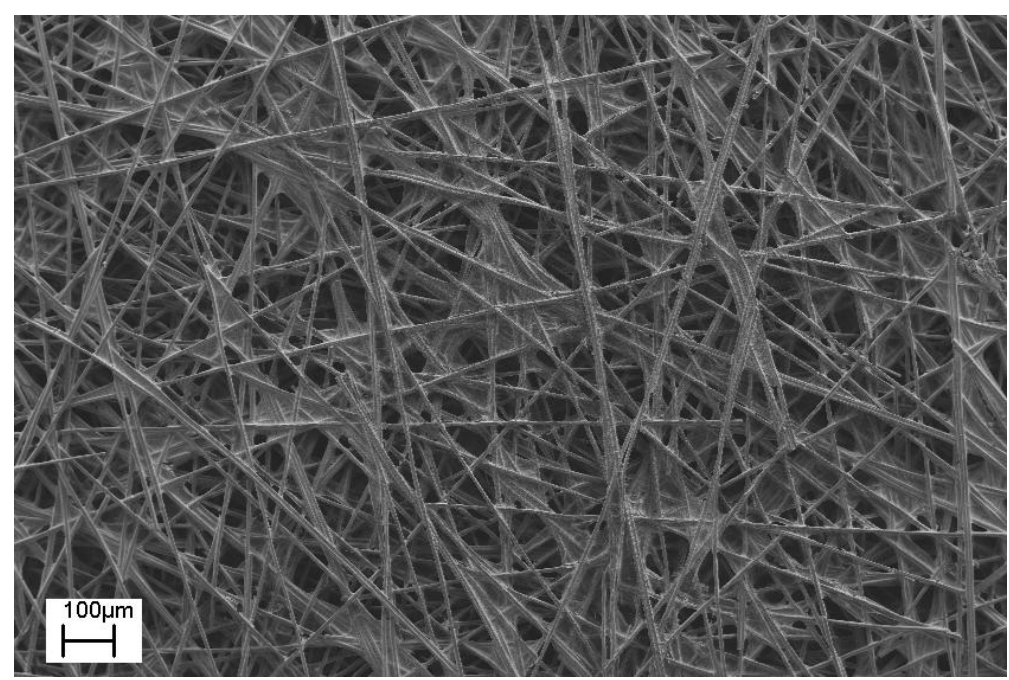

Figure 1. SEM picture of TGP 060, which is used to virtually build a virtual 3D GDL model as described in $(3,4)$.

In the model of Thiedmann et al., a SEM picture of the GDL is examined and the upper layer of fibers is identified and digitalized $(3,4)$. This data is analyzed to produce statistical equivalent layers of fibers. These layers are piled up to create a three 
dimensional geometry while the diameter of the fibers is chosen to match the size of the fibers in the original material. In the virtual version of the fibers, their perpendicular cross section is assumed to be squared rather than circular like in the original material. With this aggregation of fibers, it is possible to model a three dimensional GDL material without binder. The binder that physically connects the fibers is added in the next step like in the original material. There are different scenarios of possible binder modeling in the constructed GDL. In our study, we use the "completely filled" scenario. In this model statistically determined geometries built by crossing fibers are completely filled with binder material one after another until the porosity of the original material is reached (4).

\section{$\underline{\text { Gas-Transport Model }}$}

The gas transport through the virtual GDL is modeled with the Lattice-BoltzmannBGK method. The main part of its algorithm in its discretized form is the following equation:

$$
f_{i}\left(\vec{r}+\vec{\xi}_{i} \delta t, t+\delta t\right)-f_{i}(\vec{r}, t)=\omega \delta t\left(f_{i}^{e q}(\vec{r}, t)-f_{i}(\vec{r}, t)\right)
$$

Originally, this formula stems from the well known Boltzmann equation, a general description of gas and fluid flow set up by Ludwig Boltzmann. A derivation of Equation [1] can be found in (6).

The quantities $\mathrm{f}$ in Equation [1] relate to a number of particles high enough to guarantee continuity. For this group of particles, f may be interpreted as a function that gives the probability to find a particle at a position $r$ and a velocity $v$. The index $i$ is the discretization index for the probability function. We use a standard D3Q19 lattice (each lattice nodes has 18 neighbors in 3 dimensions). For simplicity, external forces like gravity or electromagnetic fields are neglected.

A general interpretation of the Lattice-BGK algorithm is the connection of gas transport and molecule collisions. The left side of Equation [1] describes the transport of molecules from one lattice node to its neighboring node in a time-step $\delta$ t. The velocity $\xi$ is the unit-molecule velocity whose definition guarantees that the molecules travel exactly the space discretization $\delta x$ during that time. We find the collision term on its right side, an approximation of the integral in the original Boltzmann equation assumed by Bhatnagar, Gross and Krook (8). It implies that any disequilibrium $f$ relaxes towards a local Maxwell-distribution at each time and place. Here, $\omega$ is the collision frequency and $\delta$ the time-step between two relaxations. Its first two velocity moments determine the macroscopic values of the probability function. In their analytical form, these moments are invariant under collision $(6,9)$ and the following discretized versions are equally defined for the velocity $\mathrm{u}$

$$
\rho=\sum_{i} \mathrm{f}_{\mathrm{i}}=\sum_{\mathrm{i}} \mathrm{f}_{\mathrm{i}}{ }^{\mathrm{eq}}
$$

and the density $\rho$

$$
\rho \mathrm{u}_{\alpha}=\sum_{\mathrm{i}} \xi_{\alpha, \mathrm{i}} \mathrm{f}_{\mathrm{i}}=\sum_{\mathrm{i}} \xi_{\alpha, \mathrm{i}} \mathrm{f}_{\mathrm{i}}^{\mathrm{eq}}
$$

The algorithm we use in this paper carries out a collision and a transport step based on Equation [1] and calculates new macroscopic values in each iteration loop. In the postprocess the pressure is often calculated through the pressure-density relation of gas 
kinetics $p=c_{s}^{2} \rho / 3$ (6). Another connection of the algorithm's microscopic quantities with the macroscopic input is the relation of the collision frequency $\omega$ and the viscosity $v$ of the fluid. For this relation derived in a Chapman-Enskog expansion of Equation [1] we find (6):

$$
\omega=\frac{c_{s}^{2}}{v+\delta t_{s}^{2} / 2}
$$

According to Equation [4], the higher the viscosity, the shorter the time scale $\tau=1 / \omega$ on which a fluid relaxes towards equilibrium. The isothermal speed of sound $c_{s}=\sqrt{R T}$ sets the magnitude of the molecular velocity $\xi$ in the pre-process of the simulation. Here $\mathrm{R}$ denotes the specific gas constant and $\mathrm{T}$ the temperature of the fluid. When characterizing the fluid in the following, we will mostly refer to $\omega$, which is inversely proportional to the viscosity.

\section{Characteristic numbers}

In fluid mechanics, dimensionless quantities are introduced to compare different scenarios of fluid flow. The Reynolds number is the ratio of inertial to viscous forces in a fluid, while $u_{\text {in }}$ is a reference velocity and L the macroscopic (reference) length of the material:

$$
\operatorname{Re}=\frac{\mathrm{u}_{\mathrm{in}} \mathrm{L}}{v} \approx \frac{\mathrm{Ma}}{\mathrm{Kn}}
$$

Fluids with the same Reynolds number have the same streaming behavior. This makes it possible to replace the fluid being analyzed with a physical equivalent one. When modeling fluid flow, usually numerical advantages are the reason to change the viscosity and velocity in a way that their ratio stays the same.

On the right side of Equation [5], the Mach number $\mathrm{Ma}$ is a measure for the compressibility, the Knudsen number Kn for the collision density of the fluid. With the adjustment of the velocity, the viscosity (or $\omega$ respectively) and hence Knudsen and Mach number are changed to maintain the same Reynolds number. In doing so, it is important to notice that for a discretized Boltzmann approach $\mathrm{Ma}<<1$ and $\mathrm{Kn}<<1$ should still be valid to avoid large discretization errors and unphysical results $(6,9)$. Simply varying the discretization of our lattice is another method where the same macroscopic fluid flow conditions are present. To observe both effects in our algorithm, we vary the collision frequency $\omega$ and the space discretization $\delta x$ for the same physical situation. The influence of these parameters on the physical output of a square channel is under study in this paper. Then these results are taken into account when the GDL material is analyzed and their effect on the behavior of physical quantities is observed once again.

The calculated physical quantities that help us to assess our simulation results will be the tortuosity $\mathrm{T}$ and the permeability $\kappa$. The tortuosity in its most common definition is the ratio of the flow path length through a porous material to the direct distance between the material's two ends. Since the fluid's macroscopic velocity is the quantity gained from the Lattice-Boltzmann simulation, our definition of the tortuosity is set to be

$$
\mathrm{T}=\sum_{\mathrm{i}}^{\text {lattice }}|\overrightarrow{\mathrm{u}}|_{\mathrm{i}} / \sum_{\mathrm{i}}^{\text {lattice }} \mathrm{u}_{\mathrm{x}, \mathrm{i}},
$$

in which $\mathrm{x}$ is the main flow-direction vertical to the electrode's surface. This definition we use throughout this paper. Consequently, for the gas flow through a channel without obstacles the tortuosity will be exactly 1 . 
For the permeability, we refer to the definition in (10):

$$
\kappa=\frac{\rho \mathrm{u}_{\text {lay }} v}{\Delta \mathrm{p} / \Delta \mathrm{x}}
$$

With this equation, the permeability for a fluid flow through a certain material of length $\Delta \mathrm{x}$ can be determined. In our simulation, the fluid properties density, viscosity, and the average velocity for the first layer of $\Delta x$ determine the simulated pressure on the output in Equation [7]. The permeability depends on the material properties observed but also on the main streaming direction of the gas flow. In a fuel cell like the HT-PEFC, the in-plane permeability characterizes the GDL's ability to spread the gas evenly under channels and land while the through-plane permeability determines how well the electrode is supplied with gas. In this paper, we only consider the through-plane permeability of the GDL.

A fundamental study of the viscosity dependence of the permeability can be found in (7). The viscosity dependence of the permeability for various Lattice-Boltzmann algorithms and different lattice-resolutions was observed. For the underlying geometry, Pan et al. used an accumulation of spheres. The basic idea of our paper is similar, though the inspected geometry is the GDL. In our virtual counterpart, a large 3D section of it is needed so that statistical relevance is reached. By simulating realistic macroscopic dimensions of a HT-PEFC, this requirement is fulfilled. To achieve this magnitude, we use the standard Boltzmann algorithm with simple bounce back boundaries as a first approach throughout this paper. Another difference to Pan et al. is the reference and validation we use for the permeability. In our work, we first validate our implementation for an empty square channel. The observed effects are discussed and will be compared with the corresponding effects in the virtual GDL. Finally, the results of the GDL studies are compared with experimental results for real materials.

\section{Results and Discussion}

The input variables for all our simulations are chosen according to the operating condition of a HT-PEFC: The temperature is set to $160^{\circ} \mathrm{C}$, the viscosity and density of hydrogen at this temperature are assumed for the one-component flow. We choose ZouHe boundary conditions of a homogenous velocity profile for the inlet and a uniform pressure for the outlet (11). The inlet velocity's magnitude is motivated by Faraday's law. The main flow stream is in $x$-direction in our simulations hence vertically towards the GDL. Parallel to the surface of the electrode, in y- and z-direction, wall nodes close up the ends of the channel with a square cross section. At these points, standard bounce back conditions are applied; fibers and binder of the virtual GDL are treated alike.

The discretization size is 5 lattice points for the diameter of a fiber, which corresponds to $7.5 \mu \mathrm{m}$ on the macroscopic scale. All simulations have been checked on convergence and on mass conservation.

\section{Empty Channel Analysis}

To validate our code, we simulate the gas transport for an empty square channel and compare the results with the well known analytical solution. The dimension of the channel is $1000 \times 100 \times 100$ lattice nodes, which corresponds to $1.5 \times 0.15 \times 0.15 \mathrm{~mm}$ in our discretization. The collision frequency in lattice units is set to be $\omega=1$ l.u., which determines the value for the velocity via Equation [4]. 
We monitor the pressure drop in our simulation area. Its experimental solution with the diameter $d$ of the square cross section, and an experimental constant $\varphi$ is found to be (12)

$$
\Delta \mathrm{p}_{\exp }=\frac{32 \vee \rho \mathrm{u}_{\text {lay }} \Delta \mathrm{x}}{\mathrm{d}^{2}} \varphi
$$

We calculate the average pressure for each layer of $\mathrm{x}$ in the simulation area. The reference pressure is set to be at $\mathrm{x}=500$ 1.u.. We calculate the pressure difference of the first, second, third... x-layer with the reference point and compare this result with the calculated pressure of Equation [8]. In Figure 2 we illustrate the result. The deviation from the reference value $\mathrm{p}_{\text {ref }}$ is plotted over an area of 200 lattice nodes of the coordinate in stream direction $\mathrm{x}$ for inlet (left) and outlet (right). The deviation stays at $\sim 3 \%$ for the most part of the plot and hence is in good agreement with Equation [8]. The boundary conditions cause that the profile is not fully developed on the inlet due to the fixed velocity on the boundary. This influence is noticeable up to 40 lattice sites. For the GDL simulations, this fact suggests to add an empty channel area quite large in front of the GDL geometry, the actual object under study.
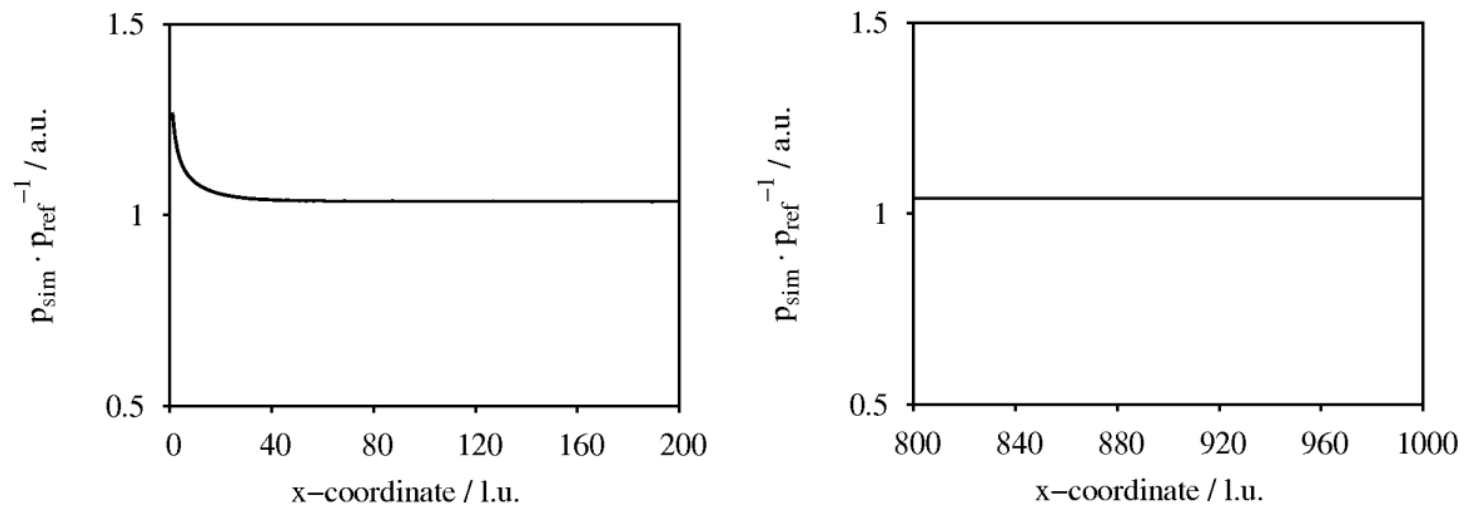

Figure 2. This Figure illustrates, how the simulated pressure for an empty channel of the size $100 \times 1000 \times 1000$ 1.u. deviates from its experimental value calculated. An area of 200 nodes in streaming direction is shown for the inlet (left) and outlet (right).

Furthermore, the tortuosity and permeability of this channel can be calculated and checked for plausibility. The tortuosity for this empty channel is $\mathrm{T}=1.0009$ and hence in very good agreement with the reference value of 1 . Basically, the full macroscopic velocity is oriented in streaming direction in the empty channel. The permeability is $\kappa=801.3 \mathrm{D}\left(\mathrm{D}=1 \mathrm{Darcy}=9.87 \times 10^{-13} \mathrm{~m}^{2}\right)$. Its error is influenced by the only simulated quantity in Equation [7], namely the pressure drop analyzed above. With more obstacles (like a GDL) in the middle of the channel, one would expect the value for the permeability to drop. As described above, we can vary the velocity and viscosity of the simulation if the Reynolds number stays the same. Substituting Equation [8] in Equation [7], we obtain a reference permeability for the empty channel: $\kappa_{\text {ref }}=800.5 \mathrm{D}$, very close to the simulated value above. The resulting equation is independent of $\rho, v$ and $\mathrm{u}$. Discretization issues and the variation of the input parameter $\omega$ may nevertheless restrict this prediction. 


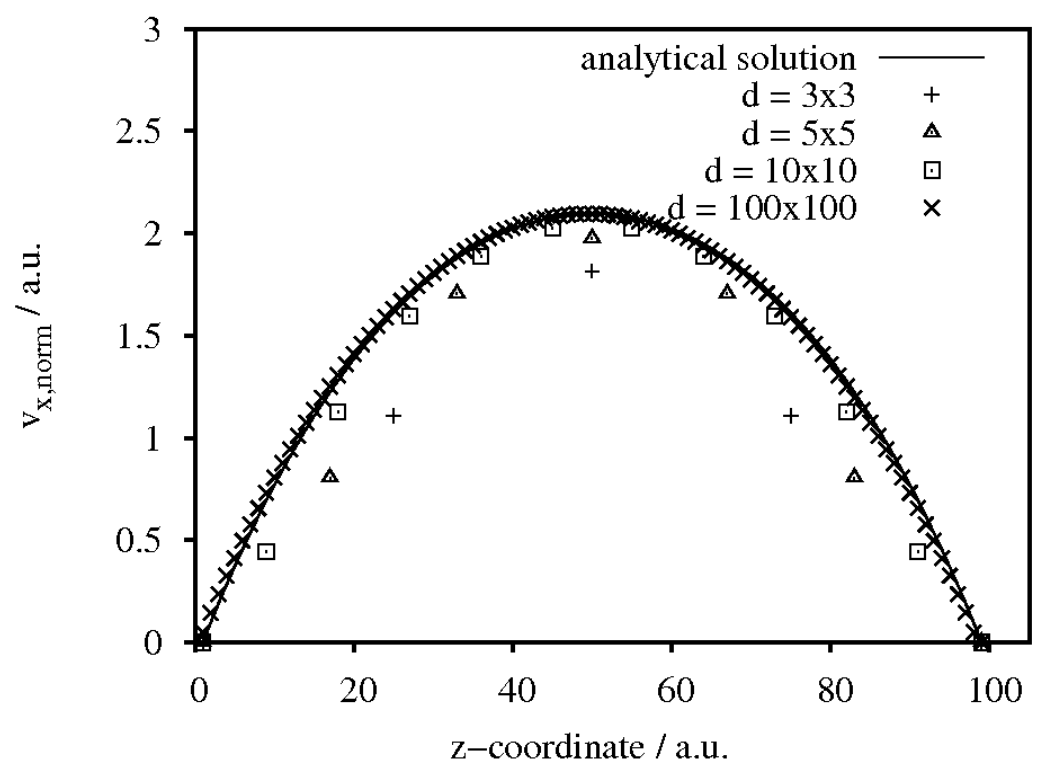

Figure 3. This figure illustrates Hagen-Poiseuille velocity profiles for an empty square channel. The analytical and simulated solution for a $1000 \times 100 \times 1001 . u$ channel are shown and, in accordance with the macroscopic geometry, various smaller discretizations with a diameter of 3, 5 and 10 l.u. In this simulation we set $\omega=1$. A $2 \mathrm{D}$ section of the 3D simulation area is shown in z-direction. The z-coordinate has been transferred to the corresponding $100 \times 100$ cross section. X- and y-coordinate are set to half the total macroscopic length.

We run the simulation of the empty channel for a various number of latticeresolutions to analyze the discretization influence. With the same macroscopic conditions as before, we simulate the fluid flow in a square channel for various discretizations. The diameter of the square tube is chosen to be 3,5 or 10 lattice nodes and $\omega$ is set to be 1 . For the qualitative examination, we plot the Hagen-Poiseuille profiles of the simulated areas and compare them with the analytical result (13). In Figure 3, 2D sections of the 3D geometry for $\mathrm{x}=0.5 \mathrm{~L}$ and $\mathrm{y}=0.5 \mathrm{~d}$ can be observed. The velocity shown on the $\mathrm{y}$-axis is normalized with the initial velocity according to its discretization. All z-coordinates of the different curves are transferred to their corresponding coordinates on a $100 \times 100$ square channel. The latter is also plotted as a reference and shows excellent agreement with the analytical result. For the smaller resolutions, a strong dependence on the discretization can be observed: The height of the profile massively decreases with lower resolution as seen in Figure 3. Hence, when $\omega$ is set to 1, the pores with a resolution of less than 5-10 lattice nodes in the GDL are expected to be responsible for velocity profile differences between simulation and analytical result.

Regarding the GDL, this means that small resolutions in narrow parts may add up to produce discretization errors in the physical output. If the velocity in these channels is too small, the pressure loss according to Equation [8] will be equally small and the permeability in Equation [7] will increase. To observe this directly, the geometry dependence of a normalized $\kappa$ is illustrated in Figure 4 . Here the results from the velocity profile are confirmed and $\kappa$ increases for smaller lattice resolution. The limit of a good agreement with its reference value depends on the choice of $\omega$. For a choice of $\omega=0.2$ 1.u. the influence is noticeable up to a cross section of $d=50$ 1.u., while for the choice of $\omega=1$ 1.u. we observe, as before, a significant dependence up to $d=10$ l.u.. We stress 
that for all simulations mass conservation is still valid. While the pressure loss in the channel decreases, its absolute value increases, and the product of velocity and density stays the same for each $\mathrm{x}$-layer when convergence is reached.

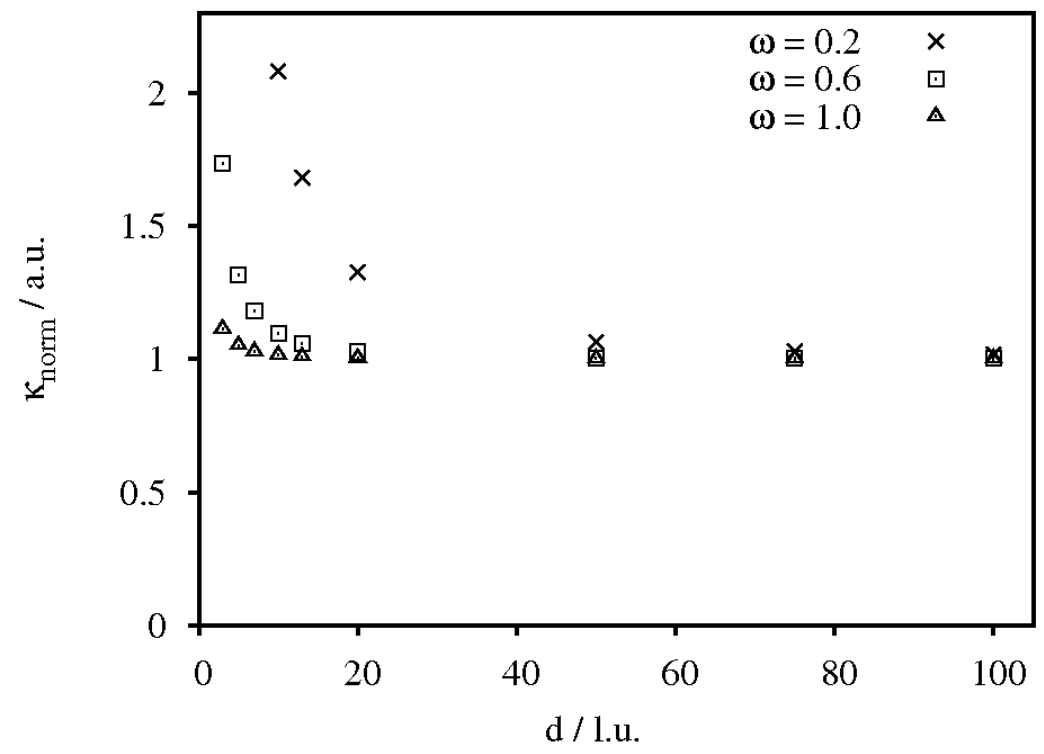

Figure 4 . The dependence of the normalized permeability $\kappa_{\text {norm }}$ on the disccretization resolution of an empty square channel are illustrated and compared. The results for the values $\omega=0.2,0.6$ and 1.0 are plotted over the diameter $\mathrm{d}$ of the square channel, which is represented in lattice units and corresponds to the same physical geometry.

We further simulate the velocity profile of the $30 \times 3 \times 3$ l.u. resolution for three different values in Figure 5 to understand its dependence of $\omega$. The simulated results illustrate the effect in the diagram. The curve for $\omega=0.21$.u. is almost the same as the planar profile set on the inlet. Since the viscosity for this curve is very high, the main force the fluid encounters are the collisions with other molecules. The influence of the bounce back boundary conditions at the wall is too small to form a profile. Again, the influence on the permeability is to be analyzed. Figure 6 shows that the influence of $\omega$ causes the permeability to raise for small resolutions. A choice $\omega>11$.u. seems to be appropriate even for the largest discretization. For $d=3 \times 3$ l.u. deviations occur over the full range of $\omega$. For a resolution of $100 \times 100$ l.u., the influence of $\omega$ is still weakly noticeable. Since the discretization for this choice is obviously large enough, a pure viscosity influence can be suspected.

As described above, two influences on the instability of permeabilities have been observed. The discretization resolutions in the empty channels have a massive effect on the physical output of the simulation. This effect heavily depends on the collision parameter chosen. To decrease the effect, our results suggest a resolution of 5-10 lattice nodes for the smallest pore space with an $\omega$ between 1 1.u. and 1.5 1.u.. As described in (7), more sophisticated choices of the LB-model and the boundary conditions should have a positive effect on these influences in return for more processing power. In the next section, we analyze this effect on the simulation of the virtual GDL material. 


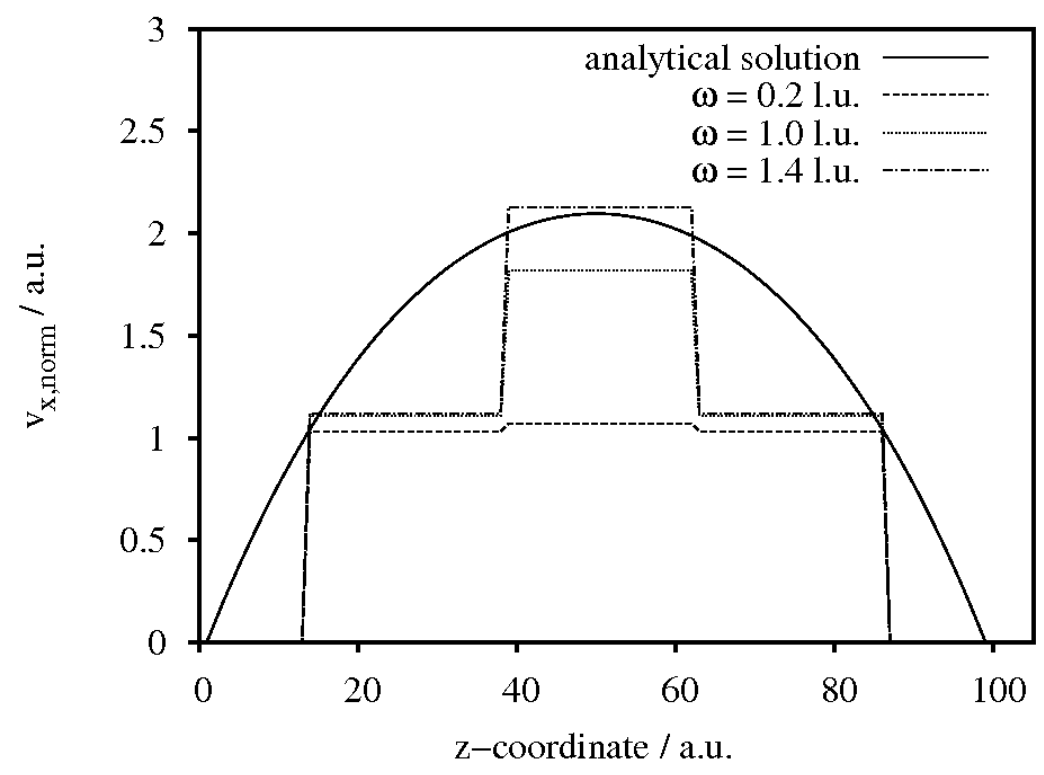

Figure 5. Hagen-Poiseuille velocity profile of an empty square channel is shown. The analytical solution is compared with a $3 \times 3$ l.u. cross section for the values $\omega=0.2,1.0$ and 1.4 1.u.. A 2D section of the 3D simulation area is shown in z-direction. The zcoordinate has been transferred to the corresponding $100 \times 100$ l.u. cross section and the points are connected in favor of clarity. X-and y-coordinate are set to half the total macroscopic length.

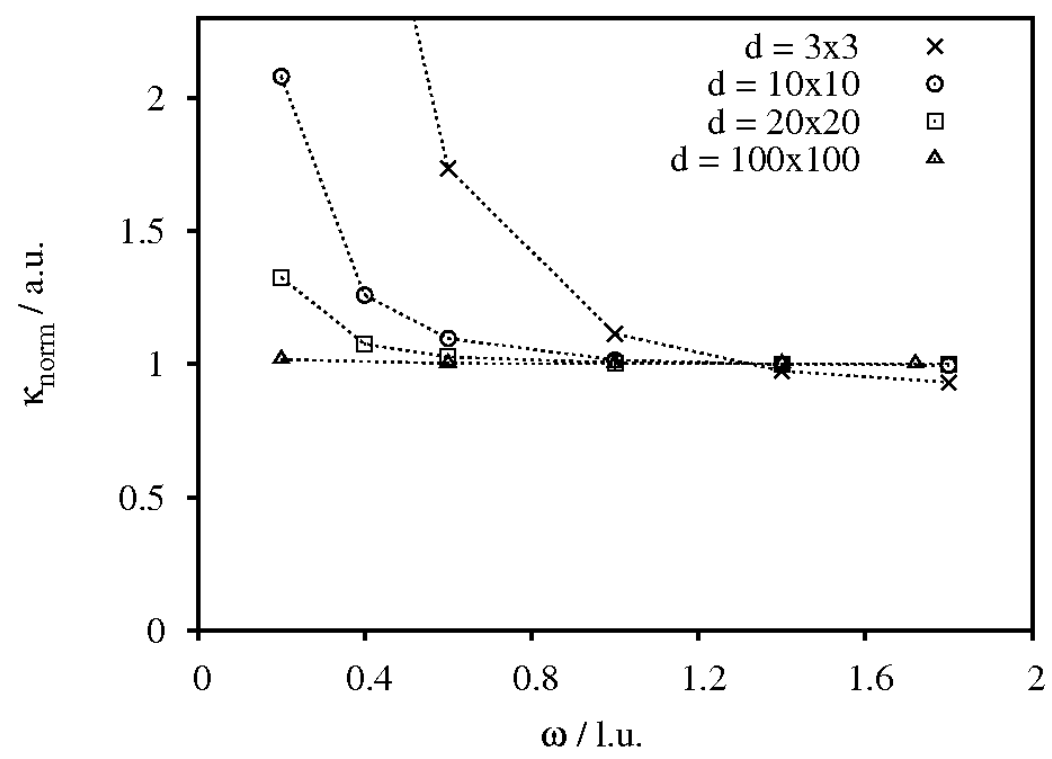

Figure 6. We simulated the gas flow in an empty square channel for various discretizations of the diameter $\mathrm{d}$. All channels correspond to the same physical size of the channel. The calculated normalized permeability $\kappa_{\text {norm }}$ is plotted over the collision frequency $\omega$ for the different discretizations. The points are connected in favor of clarity. 


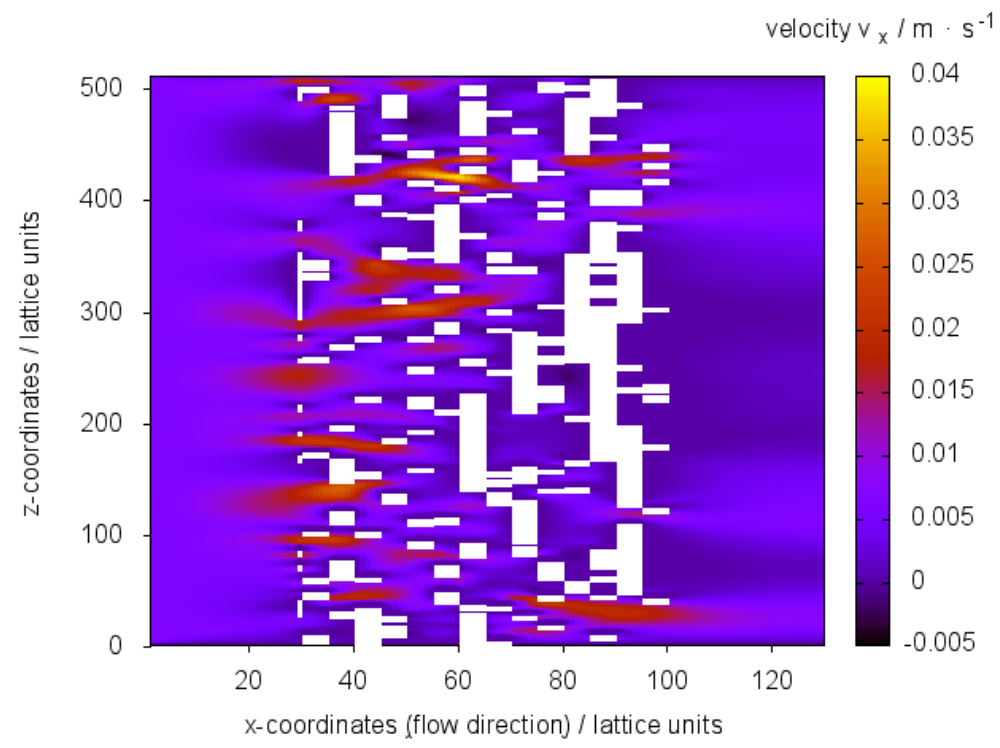

Figure 7. We illustrate a $2 \mathrm{D}$ xz-section of the simulated gas flow in a virtual GDL with a geometry of $130 \times 512 \times 512$ 1.u.. The macroscopic velocities at each lattice node are colored corresponding to their value. Binder and fiber material is colored in white.

In the HT-PEFC, the width of a channel is in the mm scale, the GDL's thickness of approximately $200 \mu \mathrm{m}$. With our choice for the discretization, a typical macroscopic GDL geometry under the channel of magnitude $0.7 \times 0.7 \times 0.1 \mathrm{~mm}$ corresponds to $512 \times 512 \times 70$ l.u. and is in the region of a the real material used in the HT-PEFC. We have added a large section of free channel on the inlet/outlet to minimize the effect of the inlet/outlet that we observed in Figure 2. In Figure 7 we exemplarily see a horizontal xzslice of the velocity distribution for the simulated region at $y=651$.u. (30 1.u. empty inlet/outlet area).

As we would expect from mass conservation, in narrow spaces the fluid velocity has its highest values. The white area at $\mathrm{x}=85 \mathrm{l}$.u. is mainly binder material that blocks the fluid at $\mathrm{x}=80$ l.u., locally redirects it and causes the velocity changes. Hence, a detailed investigation of different binder models seems to be appropriate but lies beyond the scope of this paper.

The tortuosity we obtain for the $100 \times 100$ cross section is $\mathrm{T}=1.26$ according to Equation [6]. This value does not change significantly when varying the discretization or the collision frequency and is in good agreement with (14), in which CFD simulations led to an average tortuosity of $T=1.2$ for the same virtual material. Since our main perspective during this paper is the study of parameter influences on the physical output, we will concentrate on the permeability in the following.

For the analysis of the permeability, we take the same approach as before and calculate the permeability for different choices of $\omega$ (100 l.u. empty inlet/outlet area). This dependence is shown in Figure 8. If $\omega$ gets smaller than 0.81 .u. the value of $\kappa$ massively increases. For higher $\omega$ the permeability slightly declines. This effect can also be observed for the $3 \times 3$ resolution in Figure 6. Overall, the slope of the curve resembles the one for the empty channels analyzed above. We conclude that the deviations of the permeabilities, which occur for low-resolution channels with square cross sections, and the choice of $\omega$ contribute to influence the value for the permeabilities in the GDL. 


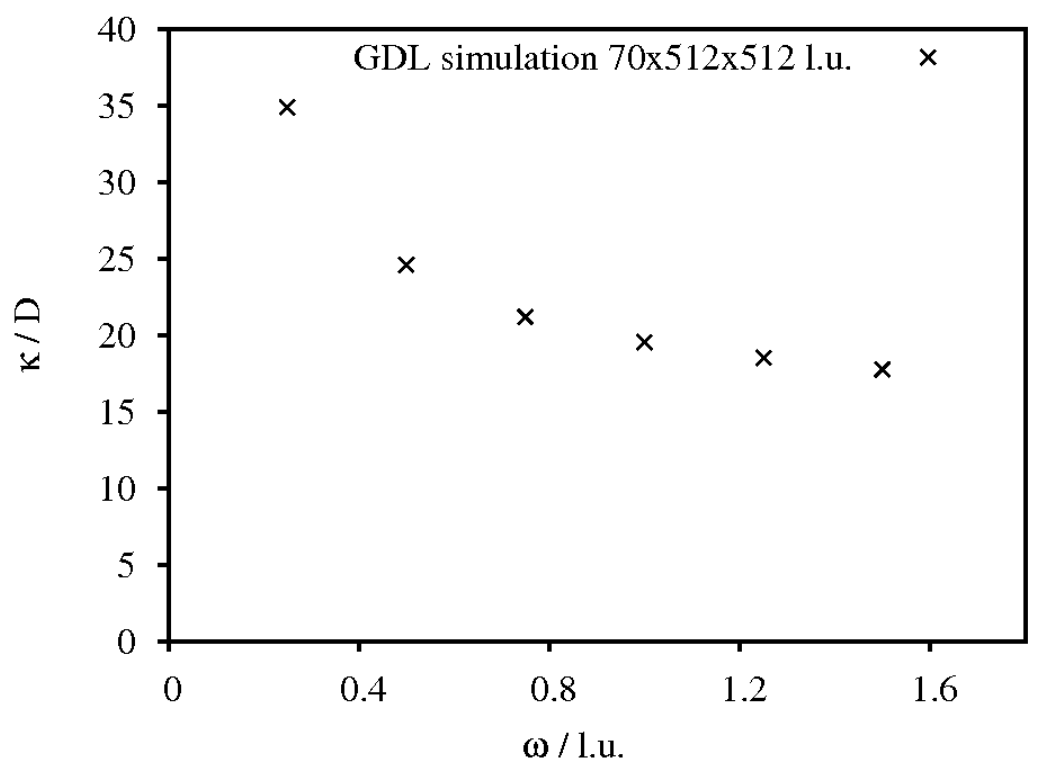

Figure 8. The gas flow through a $70 \times 512 \times 512$ l.u. channel was simulated and the calculated absolute permeabilities are plotted against the collision parameter $\omega$.

As seen above, even for highest resolutions, the choice of a small $\omega$ may cause unphysical values for $\kappa$ for the empty channels. This effect is clearly caused by the choice of $\omega$. Nevertheless, it is difficult to separate the effect caused by these parameters entirely. If the resolution gets smaller, a discretization influence for the full range of $\omega$ will be observed. This fact is also valid for the GDL simulation where low-resolution pores always contribute errors over the full range of $\omega$. In consequence the choice of perfect parameters for GDL simulations at this point is difficult to identify. Supported by the empty channel analysis and Figure 8, we observe that a choice of $\omega>0.81$.u. is advisable. An identification and variation in the number of lattice nodes in pores of the GDL will lead to a better understanding of the resolution limits in future works. Here the suggested resolution taken from Figure 3 and Figure 4 can only serve as a hint, since our modeled GDL is much more complex. We note, however, that the identification of pore spaces itself is rather difficult, especially in complex geometries. Furthermore, higher resolutions may lead to new small areas of less than 5 lattice nodes in areas of the GDL that have not yet been in solution at all before.

With this in mind, we compare our results with permeabilities from experiments. Hussaini et al. measured the permeability for air and water flow through several GDL materials according to Equation 7 (10). For the Toray TGP 060, on which the virtual GDL model is based on, they measured $\kappa=20.5$, which is in good agreement with our simulations in Figure 8.

A similar study includes another gas-component to be closer to the realistic scenario of the HT-PEFC. In this simulation, we expect further influences on permeabilities and tortuosities through the interaction-force between the different molecules. We implemented the Shan-Chen model, which contains these interaction-forces (15). As a first result, in Figure 9, we illustrate for identical components, how the velocity profile is built during our simulation. 

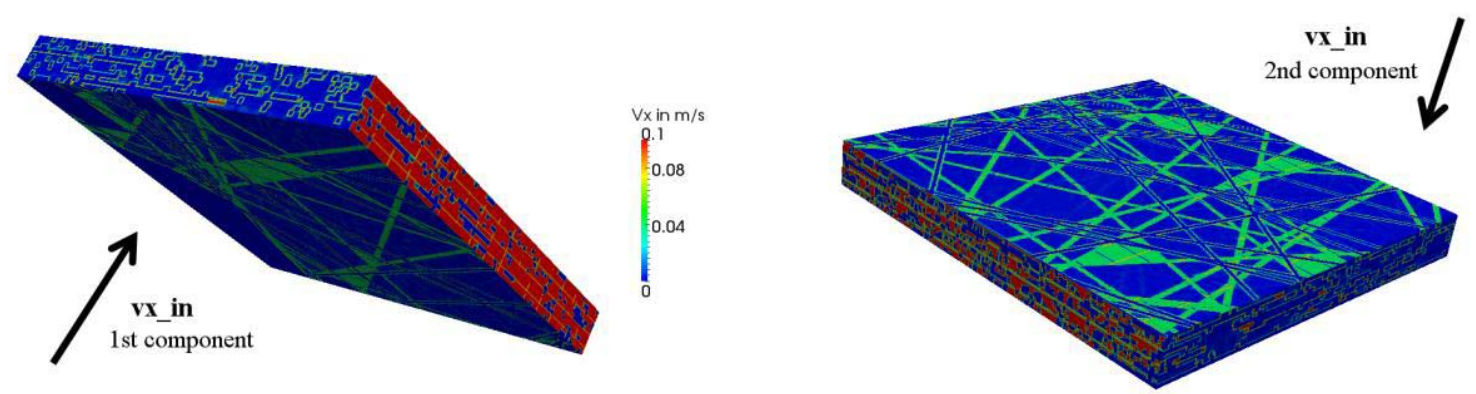

Figure 9. This figure illustrates a simulation for two component gas flow through a 70 x 512 x 512 l.u. channel. Two identical components stream from opposite directions and interact through the Shan-Chen model (15). The picture shows, how velocity profile is built during the simulation.

\section{Conclusions}

In this study, we analyzed GDL materials under the operating conditions of a HTPEM fuel cell. In order to achieve this, we combined the Lattice-Boltzmann approach with a virtual GDL material made of statistically arranged fibers. Especially, the stability of the simulated permeability and tortuosity with respect to the collision frequency and the discretization were observed. The empty channel analysis is suspected to be representative for the GDL material and the results are in accordance in many aspects.

The tortuosity is insensitive to the parameter variations. For the empty channel, the permeability shows influences of both discretization change and variation of the collision frequency, mainly for the values $\omega<11$.u. and $d<10 \delta x$. We interpret the GDL simulation results in a similar way and find a good agreement with experimental results for values of $\omega>0.8$ 1.u..

\section{Acknowledgments}

This research is funded by the German "Federal Ministry of Education and Research", within the priority program "Mathematik für Innovationen in Industrie und Dienstleistungen", grant 03MS507. Simulations are running on hardware of the Jülich Supercomputing Centre.

\section{References}

1. M. Kvesić, U. Reimer, D. Froning, L. Lüke, W. Lehnert and D. Stolten, Int. J. Hydrogen Energy, 37, 2430 (2012).

2. A. Kulikovsky, Analytical Modelling of Fuel Cells, Elsevier, Amsterdam (2010)

3. R. Thiedmann, F. Fleischer, C. Hartnig, W. Lehnert and V. Schmidt, J. Electrochem. Soc., 155(4), B391 (2008).

4. R. Thiedmann, C. Hartnig, I. Manke, V. Schmidt and W. Lehnert, J. Electrochem. Soc., 156(11), B1339 (2009)

5. D.A. Wolf-Gladrow, Lattice Gas Cellular Automata and Lattice Boltzmann Models, Springer, Berlin (2002)

6. D. Hänel, Molekulare Gasdynamik, Springer, Berlin (2004)

7. C. Pan, L. Luo and C.T. Miller, Comput. Fluids, 35, 898 (2006) 
8. P.L. Bhatnagar, E.P. Gross and M. Krook, Phys. Rev., 94, 511 (1954)

9. S. Succi, The Lattice Boltzmann Equation for Fluid Dynamics and Beyond, Clarendon Press, Oxford (2009)

10. I.S. Hussaini and C.Y. Wang, J. Power Sources, 195, 3830 (2010)

11. Q. Zou and X. He, Phys. Fluids, 9, 1591 (1997)

12. W. Kast, in VDI-Wärmeatlas: L_Druckverlust, Editor, S. Kabelac, Springer, Berlin (2006)

13. F.M White, in McGraw-Hill Series in Mechanical Engineering: Viscous Fluid Flow, J. Holman and J. Lloyd, Editors, p.120, McGraw-Hill, New York (1991)

14. Y. Wang, S. Cho, R. Thiedmann, V. Schmidt, W. Lehnert and X. Feng, Int. J. Heat Mass Transfer, 53, 2010 (1128)

15. X. Shan and H. Chen, Phys. Rev. E: Stat. Phys., Plasmas, Fluids, 47, 1815 (1993) 